\title{
Stability Analysis and Design of a Nonlinear Controller for Hot Rolling Coiler
}

\author{
Rui Li, ${ }^{1}$ Chao-nan Tong, ${ }^{1}$ and Xu Yang ${ }^{1,2}$ \\ ${ }^{1}$ School of Automation \& Electrical Engineering, University of Science and Technology Beijing, Beijing 100083, China \\ ${ }^{2}$ State Key Laboratory of Mechanical System and Vibration, Shanghai Jiao Tong University, Shanghai 200240, China \\ Correspondence should be addressed to Xu Yang; yangxu@ustb.edu.cn
}

Received 29 August 2014; Revised 21 September 2014; Accepted 21 September 2014

Academic Editor: Qingang Xiong

Copyright (c) $2015 \mathrm{Rui} \mathrm{Li}$ et al. This is an open access article distributed under the Creative Commons Attribution License, which permits unrestricted use, distribution, and reproduction in any medium, provided the original work is properly cited.

\begin{abstract}
For the new style hot rolling coiler which adopt $\mathrm{AC}$ asynchronous motor as the driving force and with using the algorithm based on differential geometry design nonlinear controller, precise coiling tension control in the rolling process of strip steel is achieved. In this paper, under the rotating orthogonal coordinate system, the fifth-order nonlinear motor model is selected as the controlled plant. By multi-input multioutput (MIMO) exact feedback linearization (EFL) algorithm, the nonlinear model is transformed to a linear one. In terms of small-gain theorem, it is the first to prove that the nonlinear coiler engine that contains the controller has characteristics of input-to-state stability. Experimental results show that the algorithm can be used for high order tracking control system with time-varying parameters. Even without the traditional flux orientation calculation, the output signals are decoupled. With this controller, the tension deviation is restricted to less than $3 \%$ and average rotational speed bias was decreased from $0.5 \%$ to $0.1 \%$ that ensure high-quality plate cut and surface of strip products.
\end{abstract}

\section{Introduction}

Constant tension is critical to uniform product thickness and smooth surface in rolling coiler. In the process of coiling, too big deviation of tension can directly lead to wrong layer, tower, loose volume, bowl volume and so forth. Through the adoption of effective control method, to improve quality of production is always the key point also the difficulty of coiling process. Because, in the process of the coiling strip, coil's diameter changed randomly, this will cause the winding speed of the coiler to change unexpectedly. In order to make the rotor speed to coincide with winding speed and the rolling tension of steel strip to keep constant, the speed of the coiler should be adjustable at any time, and speed range should be changed to adapt to the changes in coil diameter. In nearly decade, due to the improvement of hydraulic drive system and the application of advanced power electronics inverter technology, more and more AC (alternating current) motor was used as the main power of equipment coiler. High maintenance cost and complex vector control system for AC synchronous motor were compared to the complex structure. Asynchronous motor structure is relatively simple and easy to be maintained and developed. So in many giant steel mills, more asynchronous motor is implemented in technological upgrading projects in recent years [1]. Consideration of the control mode with a constant ratio of voltage frequency in general inverter and traditional regulation system in $\mathrm{AC}$ asynchronous motor is difficult to realize the coiler constant tension control. In actual system, high-speed PLC programming algorithm with the nonlinear proportional integral differential (PID) control is often adopted [2].

AC asynchronous motor contains strong coupling and high-nonlinearity factors. For the purpose of implementing a wide range precise control of the motor in the electromechanical energy conversion process, common technologies are the vector control and direct torque control. However, nonlinear control theories have not been unified. Specifically for the plants that are expressed as high order affine or nonaffine nonlinear differential equation set, the stability analysis of solving algorithm is still a hot and difficult topic which has been widely concerned. Current mainstream subjects 
about the advanced nonlinear control theorem include the passive theory, the inverse system method, the backstep approach, feedback linearization method, and active disturbance rejection control technology [3]. In the study of exact feedback linearization (EFL) for AC motor drive system, the applications in permanent magnet synchronous motor are relatively mature [4-7]. For asynchronous motor feedback exact linearization, especially for high order linearization problem of affine nonlinear systems, less detail theoretical derivation is found in the literature, for instance, the research on static two-phase coordinates of five orders linear affine nonlinear system $[8,9]$. Under the rotating coordinate system, the reduced order induction motor model with accurate linearization control is discussed $[10,11]$. But the problem of the stability analysis of an interconnected system that inferred from the theory of EFL controller has not been considered and solved yet.

This paper just chooses the classical five-order induction motor system under rotating orthogonal coordinates as the controlled plant. High order tracking controller design method of affine nonlinear system is specified. Combined with small gain theory, the problem of the stability of an interconnected high order system that inferred from the theory of EFL controller is proposed. It is proved that the nonlinear control law for Brunovsky normalized form which refers to the EFL algorithm can stabilize the overall system. This application has not been considered yet in previous literatures. Before 90s in 20th century, besides the Lyapunov method, the input to state stability (ISS) approach was a feasible tool to analyze and design the stability of complex control systems. And input to output practical stability (IOpS) as a concept was introduced by Sontag in 1989 [12]. Michel and Miller initially analyzed time-varying nonlinear interconnected system. And the small gain theory as the mathematical basis of the ISS method is defined much earlier in 1977 [13]. By the end of the 20th century, validity of small-gain approach in the ISS framework on the occasion of two interconnected subsystems was proven in Jiang et al.'s research, which stated that the general interconnection of two IOpS systems is still an IOpS system [14]. It was demonstrated that global asymptotic stability can be ensured by a nonlinear combination of partial-state feedback [15]. Based on the small gain theory and the thought of quantized nonlinear computation, it is proposed that an interconnected system is decomposed into two parts and then, respectively, proves their stability in tandem [16-18]. One part was the forward control channel structure, and the other part of homeomorphism mapping was under the new control law derived from linear feedback channel. Finally, the EFL controller of affine nonlinear systems designed can be proved to have ISS characteristics [19]. In addition, to research this complex interconnected system, some high efficient dynamic simulation approaches such as transient molecular dynamics technique have been brought in to analyze different direction currents in different modes [20, 21]. And the advanced academic ideas of adaptive particle merging/splitting and multiple/particle fluid are reasonably practical to simulate

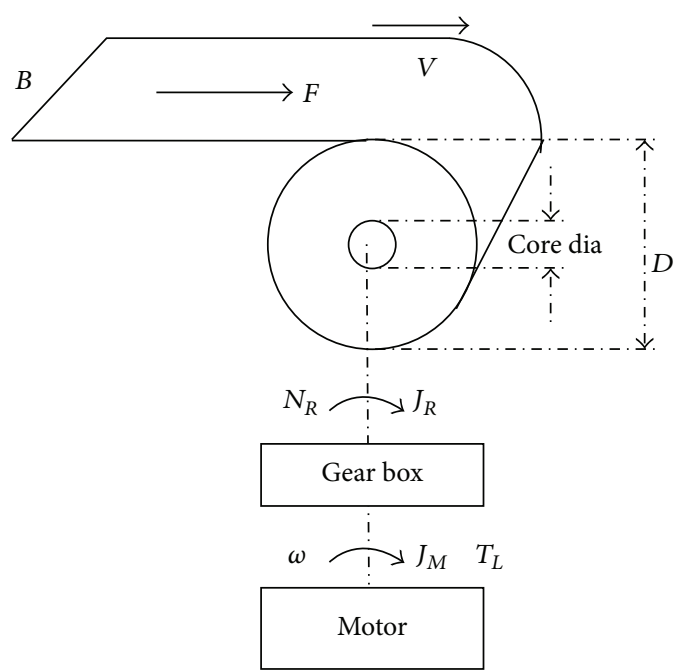

FIGURE 1: Working process of hot rolling coiler.

interconnected systems [22, 23]. Experimental results show that the method not only realizes the constant tension control but also makes the nonlinear system dynamic characteristics steady and fast. When the load of asynchronous motor has changed during a wide range, the control law based on the differential geometry method can guarantee the stable operation of the affine nonlinear systems, in which internal mutual coupling dynamic systems are included.

\section{Dynamic Control Model of Asynchronous Motor in Coiler}

This indirect coiler tension control method makes full use of the armature current of the motor which is directly proportional to coil tension that is just proportional to the diameter of coiling block. So we adopted the real-time measurement of the size of coil diameter as feedback signal, by controlling the motor armature current and excitation flux; a constant indirect tension control can be achieved. In the diagram of working process of hot rolling coiler, as is shown in Figure 1, in the process of coiling, the model of plate strip tension is established according to the coiling machine mechanical structure and transmission characteristics [24].

In Figure $1, F$ is rolling tension, $T_{L}$ is the motor load torque, $V$ is speed of the horizontal motion of the strip steel, $N_{R}$ is rotating speed of the rolling drum via slow gear box, $\omega$ is the output angular velocity of motor, $B$ is width of plate strip, $D$ is a coil diameter, and $J$ total motor inertia, $J=J_{M}+J_{R}$, where the $J_{M}$ inertia of the rotor, $J_{R}=J_{R 1}+J_{R 2}+J_{R 3}$, presents rotational inertia of the drum, including $J_{R 1}$ inertia of scrolls, $J_{R 2}$ inertia of coil steel, and $J_{R 3}$ inertia of gears.

In the process of coiling, coil diameter $D_{t}$ changed every moment. In order to keep a good shape, requirement for moving strip steel is to keep tension constant of coiling in horizontal direction. The motor's multiple parameters must follow the change of roll diameter and make dynamic adjustment real time. For a certain type of plate strip product, 
torque and coiler diameter have the following certain relationship [25]:

$$
T_{L}=F \cdot \frac{D_{t}}{2} \cdot \frac{1}{k}
$$

In formula (1), $T_{L}$ is the torque attached to the motor shaft, $D_{t}$ is instantaneous coil diameter, $F$ is the tension under plate strip coiling, and $k$ is reduction ratio of winding roll. $T_{e}$ is electromagnetic torque produced by the winding motor, and excitation flux $\Phi$ and the armature current $I$ have the following relationship:

$$
T_{e}=C_{M} \cdot \Phi \cdot I
$$

In formula (2), $C_{M}$ is constant of winding motor, which is associated with motor structure. $\Phi$ is excitation flux of winding. $I$ is current in armature winding.

For three-phase asynchronous motor as main drive of coiler, the dynamic state space model contains flux equations, voltage equations, torque equation, and the equation of motion. There is the following hypothesis.

(a) Three-phase winding is considered as symmetrical. Ignore the space harmonic and the magnetomotive force distribution in air gap according to the sine law.

(b) Ignore the saturation in magnetic circuit. The winding self-inductance and mutual inductance are constant.

(c) Ignore the power loss in iron core.

(d) In the winding resistance, the little influence from changes of frequency and temperature is regardless.

When the power of AC asynchronous motor is supplied by PWM drive circuit and the inverter, its control input is stator voltage in the three-phase winding. Through $3 / 2$ transformation and rotation transformation, the voltage equations of stator and rotor were changed to arbitrary rotation coordinate system. The dynamic mathematical model of asynchronous motor is deduced.

The system state space variable is

$$
\begin{aligned}
\mathbf{x} & =\left[\begin{array}{lllll}
x_{1} & x_{2} & x_{3} & x_{4} & x_{5}
\end{array}\right]^{T} \\
& =\left[\begin{array}{lllll}
\omega & \psi_{s d} & \psi_{s q} & i_{s d} & i_{s q}
\end{array}\right]^{T} .
\end{aligned}
$$

The system input variable is

$$
\begin{aligned}
\mathbf{u} & =\left[\begin{array}{llll}
u_{1} & u_{2} & u_{3} & u_{4}
\end{array}\right]^{T} \\
& =\left[\begin{array}{llll}
u_{s d} & u_{s q} & \omega_{1} & T_{L}
\end{array}\right]^{T} .
\end{aligned}
$$

State space equation of AC asynchronous motor system is

$$
\begin{gathered}
\frac{d \omega}{d t}=\frac{n_{p}^{2}}{J}\left(i_{s q} \psi_{s d}-i_{s d} \psi_{s q}\right)-\frac{n_{p}}{J} T_{L}, \\
\frac{d \psi_{s d}}{d t}=-R_{s} i_{s d}+\omega_{1} \psi_{s q}+u_{s d}, \\
\frac{d \psi_{s q}}{d t}=-R_{s} i_{s q}-\omega_{1} \psi_{s d}+u_{s q}, \\
\frac{d i_{s d}}{d t}=\frac{1}{\sigma L_{s} T_{r}} \psi_{s d}+\frac{1}{\sigma L_{s}} \omega \psi_{s q}-\frac{R_{s} L_{r}+R_{r} L_{s}}{\sigma L_{s} L_{r}} i_{s d} \\
+\left(\omega_{1}-\omega\right) i_{s q}+\frac{u_{s d}}{\sigma L_{s}}, \\
\frac{d i_{s q}}{d t}=\frac{1}{\sigma L_{s} T_{r}} \psi_{s q}-\frac{1}{\sigma L_{s}} \omega \psi_{s d}-\frac{R_{s} L_{r}+R_{r} L_{s}}{\sigma L_{s} L_{r}} i_{s q} \\
-\left(\omega_{1}-\omega\right) i_{s d}+\frac{u_{s q}}{\sigma L_{s}} .
\end{gathered}
$$

And the system output function is

$$
\mathbf{y}=\left[\begin{array}{ll}
h_{1}(x) & h_{2}(x)
\end{array}\right]^{T}=\left[\begin{array}{ll}
\omega & \psi_{s}
\end{array}\right]^{T}=\left[\begin{array}{ll}
\omega & \sqrt{\psi_{s d}^{2}+\psi_{s q}^{2}}
\end{array}\right]^{T} .
$$

In formula (5), $R, i, \psi, u, L, M$ represent the flux linkage, the input resistance, current, flux, voltage, inductance, and mutual inductance, respectively. The characters " $d$ " and " $q$ " note two-phase rotating coordinates as $d$-axis and $q$-axis. " $d$ " and " $r$," respectively, express on behalf of stator and rotor. Respectively, $J, n_{p}, \omega, \omega_{1}$, and $T_{L}$ is the inertia of the rotor, number of poles, the angular velocity of the mechanical rotor, the rotating angular velocity of $d_{-} q$ coordinate system, and the output mechanical torque. The rotor time constant is $T_{r}=L_{r} / R_{r}$; magnetic flux leakage coefficient of motor is $\sigma=1-L_{m}^{2} / L_{s} L_{r}:$

$$
\frac{J}{n_{p}} \frac{d \omega}{d t}=T_{e}-T_{L}
$$

Electromagnetic torque output is

$$
\begin{aligned}
T_{e} & =n_{p}\left(i_{s q} \psi_{s d}-L_{s} i_{s d} i_{s q}-i_{s d} \psi_{s q}+L_{s} i_{s q} i_{s d}\right) \\
& =n_{p}\left(i_{s q} \psi_{s d}-i_{s d} \psi_{s q}\right) .
\end{aligned}
$$

Consider that $\omega_{1}$ and $T_{L}$ are physical quantities that can be measurable real time, so they can be treated as known parameters. Then we can reduce dimension of the input vector.

\section{Exact Feedback Linearization Algorithm and Regulator Design}

To facilitate the theoretical derivation, simplify the asynchronous motor affine nonlinear system model described by formulas (5) and (6) as

$$
\begin{gathered}
\dot{\mathbf{x}}=\mathbf{f}(\mathbf{x})+\mathbf{g}(\mathbf{x}) \mathbf{u}, \\
\mathbf{y}=\mathbf{h}(\mathbf{x}),
\end{gathered}
$$


where

$$
\begin{gathered}
\mathbf{f}(\mathbf{x})=\left[\begin{array}{c}
A\left(x_{2} x_{5}-x_{3} x_{4}\right)-B \\
C x_{3}-D x_{4} \\
-C x_{2}-D x_{5} \\
C x_{5}+E x_{2}+G x_{1} x_{3}-F x_{4}-x_{1} x_{5} \\
-C x_{4}+E x_{3}-G x_{1} x_{2}-F x_{5}+x_{1} x_{4}
\end{array}\right], \\
\mathbf{g}(\mathbf{x})=\left[\begin{array}{ll}
0 & 0 \\
1 & 0 \\
0 & 1 \\
G & 0 \\
0 & G
\end{array}\right] \\
\mathbf{y}=\mathbf{h}(\mathbf{x})=\left[\begin{array}{c}
h_{1}(\mathbf{x}) \\
h_{2}(\mathbf{x})
\end{array}\right]=\left[\begin{array}{c}
x_{1} \\
\sqrt{x_{2}^{2}+x_{3}^{2}}
\end{array}\right]
\end{gathered}
$$

and $A=n_{p}^{2} / J, B=\left(n_{p} / J\right) T_{L}, C=\omega_{1}, D=R_{s}, E=1 / \sigma L_{s} T_{r}$, $F=\left(R_{s} L_{r}+R_{r} L_{s}\right) / \sigma L_{s} L_{r}$, and $G=1 / \sigma L_{s}$.

Design controller of the MIMO nonlinear system based on EFL algorithm for affine nonlinear system is divided into six steps below.

Step 1. Obtain the Child relation degree of the output function in terms of the state function. By model of formula (9), we get

$$
\begin{aligned}
& L_{g 1} L_{f}^{0} h_{1}(x)=0, \\
& L_{g 2} L_{f}^{0} h_{1}(x)=0, \\
& L_{g 1} L_{f}^{1} h_{1}(x)=A x_{5}-A G x_{3}, \\
& L_{g 2} L_{f}^{1} h_{1}(x)=A G x_{2}-A x_{4} .
\end{aligned}
$$

When $x_{5} \neq G x_{3}$ or $x_{4} \neq G x_{2}$, Lie derivatives of $h_{1}(x)$ relative to the inputs are not all 0 , so $r_{1}=k+1=2$.

Similarly,

$$
\begin{aligned}
& L_{g 1} L_{f}^{0} h_{2}(x)=x_{2}\left(x_{2}^{2}+x_{3}^{2}\right)^{-1 / 2}, \\
& L_{g 2} L_{f}^{0} h_{2}(x)=x_{3}\left(x_{2}^{2}+x_{3}^{2}\right)^{-1 / 2} .
\end{aligned}
$$

When $x_{2} \neq 0$ or $x_{3} \neq 0$, Lie derivatives of $h_{2}(x)$ relative to the inputs are not all 0 , so $r_{2}=1$.

In summary, when $i_{s d} \neq 0$ and $L_{m}^{2} \neq L_{s} L_{r}$,

$$
\mathbf{B}(x)=\left[\begin{array}{ll}
L_{g 1} L_{f}^{r_{1}-1} h_{1}(x) & L_{g 2} L_{f}^{r_{1}-1} h_{1}(x) \\
L_{g 2} L_{f}^{r_{2}-1} h_{2}(x) & L_{g 2} L_{f}^{r_{2}-1} h_{2}(x)
\end{array}\right],
$$

is nonsingular matrix, and we get the relation degree set of original system: $r=\left\{r_{1}, r_{2}\right\}=\{2,1\}$. In this case, $r<n$; therefore, the system should be further investigated whether the exact linearization exists.

Theorem 1 (see [10]). For MIMO affine nonlinear system $\dot{\mathbf{x}}=$ $\mathbf{f}(\mathbf{x})+\sum_{i=1}^{m} \mathbf{g}_{i}(\mathbf{x}) \mathbf{u}_{i}$, choose indicator values $m=n_{1} \geq n_{2} \geq$ $\cdots n_{N}, \sum_{i=1}^{N} n_{i}=n$, where $n=5$ is system dimension.

It could be exact linearized as if the system can meet the following two conditions:

(1) $D_{n}=\left[g_{1} \cdots g_{n_{1}}, a d_{f} g_{1} \cdots a d_{f} g_{n_{2}}, a d_{f}^{N-1} g_{1} \cdots\right.$ $\left.a d_{f}^{N-1} g_{n_{N}}\right]$ is nonsingular in definition domain;

(2) in groups of sets for each vector field,

$$
\begin{aligned}
D_{1}= & \left\{g_{1}\right\} \cdots D_{n_{1}}=\left\{g_{1} \cdots g_{n_{1}}\right\}, \\
& \vdots \\
D_{n_{1}+1}= & \left\{D_{n_{1}} ; a d_{f} g_{1}\right\} \cdots D_{n_{1}+n_{2}}=\left\{D_{n_{1}} ; a d_{f} g_{1} \cdots a d_{f} g_{n_{2}}\right\}, \\
& \vdots \\
D_{n}= & \left\{D_{n-n_{N}} ; a d_{f}^{N-1} g_{1} \cdots a d_{f}^{N-1} g_{n_{N}}\right\} .
\end{aligned}
$$

Every vector field in these groups of sets must be involutory.

Consider the system. The order $n=5$, the number of control inputs $m=2$. As $n_{1} \geq n_{2} \geq n_{3}=2, \sum_{i=1}^{N} n_{i}=n$, choose indicators $n_{1}=m=2, n_{2}=2, n_{3}=1$; that is, $N=3$. According to Theorem 1, constitute vector field sets:

$$
\begin{aligned}
& D_{1}=\left\{g_{1}\right\}, \\
& D_{2}=D_{m}=\left\{g_{1}, g_{2}\right\}, \\
& D_{3}=D_{m+1}=\left\{g_{1}, g_{2}, a d_{f} g_{1}\right\}, \\
& D_{4}=D_{m+n_{2}}=\left\{g_{1}, g_{2}, a d_{f} g_{1}, a d_{f} g_{2}\right\}, \\
& D_{5}=D_{m+n_{2}+n_{3}}=\left\{g_{1}, g_{2}, a d_{f} g_{1}, a d_{f} g_{2}, a d_{f}^{2} g_{1}\right\} .
\end{aligned}
$$

By calculating, $D_{1} \cdots D_{5}$ are all involutory. Now to examine whether matrix $\mathbf{D}$ is nonsingular,

$$
\begin{aligned}
\mathbf{D}_{n \times n} & =\left[g_{1}, g_{2}, a d_{f} g_{1}, a d_{f} g_{2}, a d_{f}^{2} g_{1}\right] \\
& =\left[\begin{array}{ccccc}
0 & 0 & A G x_{3}-A x_{5} & A\left(x_{4}-G x_{2}\right) & A\left(2 C x_{4}-2 E x_{3}+F x_{5}-x_{1} x_{4}-2 C G x_{2}-2 D G x_{5}+F G x_{3}+G x_{1} x_{2}\right) \\
1 & 0 & D G & -C & -C^{2}-D(E-F G) \\
0 & 1 & C & D G & 2 C D G \\
G & 0 & F G-E & -C G & 2 A G x_{3} x_{5}-C^{2} G-A x_{5}^{2}-A G^{2} x_{3}^{2}-D E G-F(E-F G) \\
0 & G & C G & F G-E & \left(A x_{5}-A G x_{3}\right)\left(x_{4}-G x_{2}\right)-\left(C-x_{1}\right)(E-F G)-C E+C F G+D G^{2} x_{1}
\end{array}\right] .
\end{aligned}
$$


After elementary transformation: $\mathbf{D}_{n \times n} \rightarrow \mathbf{I}_{5}, \mathbf{D}_{n \times n}$ is of full rank. According to Theorem 1, the original affine nonlinear system could be exact linearized.

Step 2. Recursive calculation of the linear vector field. $\bar{D}_{i} \in$ $D_{i}(x), i=1, \ldots, n$. Consider $\bar{D}_{i}$ as simple as possible. And then it can be chosen as

$$
\bar{D}=\left[\begin{array}{ccccc}
-1 & 1 & 1 & 1 & 1 \\
1 & -1 & 1 & 1 & 1 \\
1 & 1 & -1 & 1 & 1 \\
1 & 1 & 1 & -1 & 1 \\
1 & 1 & 1 & 1 & -1
\end{array}\right] .
$$

According to function,

$$
\begin{aligned}
\bar{D}_{n}+ & \sum_{j=1}^{m} k_{j}^{(n)}(x) g_{j}(x)+\sum_{j=1}^{n_{2}} k_{m+j}^{(n)}(x) a d_{f} g_{j}(x) \\
& +\cdots+\sum_{j=1}^{n_{N}} k_{m+\cdots n_{N-1}+j}^{(n)}(x) a d_{f}^{N-1} g_{j}(x)=0 .
\end{aligned}
$$

Set

$$
\begin{aligned}
& k_{1}^{(2)}=x_{1} \cdot k_{1}^{(3)}=x_{1}, \quad k_{2}^{(3)}=x_{4} \cdot k_{1}^{(4)}=x_{1}, \quad k_{2}^{(4)}=x_{4}, \\
& k_{3}^{(4)}=x_{5} \cdot k_{1}^{(5)}=x_{1}, \quad k_{2}^{(5)}=x_{1}, \quad k_{3}^{(5)}=x_{1} k_{4}^{(5)}=x_{1} .
\end{aligned}
$$

Then, we get

$$
\begin{aligned}
& k_{1}^{(2)}=-\frac{\bar{D}_{1}}{g_{1}}, \\
& k_{2}^{(2)}=-\frac{\left(\bar{D}_{2}+k_{1}^{(2)} g_{1}\right)}{g_{2}}, \\
& k_{3}^{(3)}=-\frac{\left(\bar{D}_{3}+k_{1}^{(3)} g_{1}+k_{2}^{(3)} g_{2}\right)}{a d_{f} g_{1}}, \\
& k_{4}^{(4)}=-\frac{\left(\bar{D}_{4}+k_{1}^{(4)} g_{1}+k_{2}^{(4)} g_{2}+k_{3}^{(4)} a d_{f} g_{1}\right)}{a d_{f} g_{2}}, \\
& k_{5}^{(5)}=-\frac{\left(\bar{D}_{5}+k_{1}^{(5)} g_{1}+k_{2}^{(5)} g_{2}+k_{3}^{(5)} a d_{f} g_{1}+k_{4}^{(5)} a d_{f} g_{2}\right)}{a d_{f}^{2} g_{1}} .
\end{aligned}
$$

Step 3. Solve the mapvping between different coordinate systems.

Theorem 2 (Frobenius theorem [26]). Let vector field $Y_{1}(x)$, $Y_{2}(x) \cdots Y_{k}(x)$ be already known. Dimension of $x$ is n. According to the function $h(x)$, partial differential equations are defined in space as $(\partial h(x) / \partial x)\left[Y_{1}(x) Y_{2}(x) \cdots Y_{k}(x)\right]=0$, $k<n$. At the point $x=x^{0}$, If and only if the rank of $\left[Y_{1}(x) Y_{2}(x) \cdots Y_{k}(x)\left[Y_{i}, Y_{j}\right]\right]$ at $x=x^{0}$ is still $k$, the matrix is augmented matrix including $\left[Y_{i}, Y_{j}\right]$ the Lie bracket of any twocolumn vector of matrix $\mathrm{Y}$. There must be $(n-k)$ scalar function $h_{1}(x), h_{2}(x), \ldots, h_{n-k}(x)$ defined in field of $x^{0}$, which is solution of the partial differential equations system. And the rank of Jacobian matrix $\mathbf{J}\left[h_{1}(x) h_{2}(x) \cdots h_{n-k}(x)\right]^{T}$ is $(n-k)$.

By the integral curve obtained in the vector field $\bar{D}_{1} \cdots \bar{D}_{n}$, the relationship mapping is deduced in state space $\mathbb{R}^{n}$ that is expressed as a new coordinate " $w$ " from the original coordinate $\mathbf{x}$ :

$$
\mathbf{F}\left(w_{1}, \ldots, w_{n}\right)=\Phi_{w_{1}}^{\bar{D}_{1}} \ldots \ldots \circ \Phi_{w_{n}}^{\bar{D}_{n}}(\mathbf{x}) .
$$

Select an arbitrary point in the domain, and start on the vector $\bar{D}_{n}$; recursive relation can be obtained from the differential equation: $\left(d / d w_{n-1}\right)\left[x_{1}, \ldots, x_{n}\right]^{T}=\bar{D}_{n-1}, x(0)=$ $\Phi_{w_{n}}^{\bar{D}_{n}}$. According to Theorem 2, this differential equation is solvable. Finally by formula (21), we get the required mapping $\mathbf{x}=\mathbf{F}(\mathbf{w})$ :

$$
\begin{aligned}
& x_{1}=w_{1}+w_{2}+w_{3}+w_{4}-w_{5}, \\
& x_{2}=w_{1}+w_{2}+w_{3}-w_{4}+w_{5}-1, \\
& x_{3}=w_{1}+w_{2}-w_{3}+w_{4}+w_{5}+1, \\
& x_{4}=w_{1}-w_{2}+w_{3}+w_{4}+w_{5}-1, \\
& x_{5}=w_{2}+w_{3}+w_{4}+w_{5}+1 .
\end{aligned}
$$

Get the inverse mapping of formula (22) which is

$$
\begin{aligned}
& w_{1}=x_{1}-x_{5}+1, \\
& w_{2}=\frac{x_{1}}{2}-\frac{x_{4}}{2}-\frac{1}{2}, \\
& w_{3}=\frac{x_{1}}{2}-\frac{x_{3}}{2}+\frac{1}{2}, \\
& w_{4}=\frac{x_{1}}{2}-\frac{x_{2}}{2}-\frac{1}{2}, \\
& w_{5}=\frac{3 x_{1}}{2}-\frac{x_{2}}{2}-\frac{x_{3}}{2}-\frac{x_{4}}{2}-\frac{x_{5}+1}{2} .
\end{aligned}
$$

Step 4. Calculate the induced mapping. Based on the space mapping above, the induced mapping from the original system $\mathbf{f}(\mathbf{x})$ is obtained as $\mathbf{F}_{*}^{-1}(\mathbf{x})=\mathbf{J}_{\mathrm{F}^{-1}} \mathbf{f}(\mathbf{x})$ and then put $\mathbf{x}=\mathbf{F}(\mathbf{w})$ in the mapping relationship, and note $f^{(0)}(w)$. 
$\mathbf{J}_{\mathbf{F}^{-1}}$ is the Jacobian matrix of $\mathbf{F}^{-1}(\mathbf{x})$ :

$$
f^{(0)}(w)
$$

$$
=\left[\begin{array}{c}
C x_{4}-B-E x_{3}+F x_{5}-x_{1}\left(x_{4}-G x_{2}\right)+A x_{2} x_{5}-A x_{3} x_{4} \\
\frac{F x_{4}}{2}-\frac{C x_{5}}{2}-\frac{E x_{2}}{2}-\frac{B}{2}+x_{1}\left(\frac{x_{5}}{2}-\frac{G x_{3}}{2}\right)+\frac{A x_{2} x_{5}}{2}-\frac{A x_{3} x_{4}}{2} \\
\frac{C x_{2}}{2}-\frac{B}{2}+\frac{D x_{5}}{2}+\frac{A x_{2} x_{5}}{2}-\frac{A x_{3} x_{4}}{2} \\
\frac{D x_{4}}{2}-\frac{C x_{3}}{2}-\frac{B}{2}+\frac{A x_{2} x_{5}}{2}-\frac{\left(A x_{3} x_{4}\right)}{2} \\
\frac{\left(C x_{2}\right)}{2}-\frac{3 B}{2}-\frac{C x_{3}}{2}+C x_{4}-\frac{C x_{5}}{2}+\frac{D x_{4}}{2}+\frac{D x_{5}}{2}-\frac{E x_{2}}{2}-E x_{3}+\frac{F x_{4}}{2}+F x_{5}-x_{1}\left(x_{4}-\frac{x_{5}}{2}-G x_{2}+\frac{G x_{3}}{2}\right)+\frac{3 A x_{2} x_{5}}{2}-\frac{3 A x_{3} x_{4}}{2}
\end{array}\right]
$$

For achieving the last transformation of state feedback, define medium transformation function which is $\mathbf{R}_{j}, j=1, \ldots, N-$ 1 ,

$\mathbf{R}_{1}$

$$
\left[\begin{array}{c}
\frac{C x_{2}}{2}-\frac{B}{2}+\frac{D x_{5}}{2}+\frac{A x_{2} x_{5}}{2}-\frac{A x_{3} x_{4}}{2} \\
\frac{D x_{4}}{2}-\frac{C x_{3}}{2}-\frac{B}{2}+\frac{A x_{2} x_{5}}{2}-\frac{A x_{3} x_{4}}{2} \\
\frac{C x_{2}}{2}-\frac{3 B}{2}-\frac{C x_{3}}{2}+C x_{4}-\frac{C x_{5}}{2}+\frac{D x_{4}}{2}+\frac{D x_{5}}{2}-\frac{E x_{2}}{2}-E x_{3}+\frac{F x_{4}}{2}+F x_{5}-x_{1}\left(x_{4}-\frac{x_{5}}{2}-G x_{2}+\frac{G x_{3}}{2}\right)+\frac{3 A x_{2} x_{5}}{2}-\frac{3 A x_{3} x_{4}}{2} \\
\frac{x_{1}}{2}-\frac{x_{2}}{2}-\frac{1}{2} \\
\frac{3 x_{1}}{2}-\frac{x_{2}}{2}-\frac{x_{3}}{2}-\frac{x_{4}}{2}-\frac{x_{5}+1}{2}
\end{array}\right] .
$$

$$
\mathbf{z}_{i}^{(j)}= \begin{cases}f_{n_{j}+i}^{(j-1)}(\mathbf{w}) & i=1, \ldots, n-n_{j}, \\ z_{i}^{(j-1)}=w_{i} & i=n-n_{j}+1, \ldots, n\end{cases}
$$

where $f^{(j)}(\mathbf{w})=\mathbf{J}_{\mathbf{R}_{j}}(\mathbf{w}) f^{(j-1)}(\mathbf{w})$.

By $f^{(0)}(w)$ and formula (24), via recursion relation of (25), we have
Similarly, we get $\mathbf{R}_{N-1}$. As $N=3, \mathbf{R}_{2}$ is the coordinate transformation from space $w$ to space $z$, which is what we acquired. Define composite transformation $\left.\mathbf{T} \triangleq \mathbf{R}_{N-1} \mathbf{F}^{-1}\right|_{w=\mathbf{F}^{-1}(\mathbf{x})}$. Under this composite transformation, vector field transformation of the original system is $\widetilde{\mathbf{f}}(\mathbf{x})$ and $\widetilde{\mathbf{g}}(\mathbf{x})$; that is, $\widetilde{\mathbf{f}}(\mathbf{x})=$ $\mathbf{J}_{T}(\mathbf{x}) \mathbf{f}(\mathbf{x}), \widetilde{\mathbf{g}}(\mathbf{x})=\mathbf{J}_{T}(\mathbf{x}) \mathbf{g}(\mathbf{x})$.

Step 5. Obtain the Brunovsky normalized form and nonlinear control law. The transformation of calculated coordinates above is denoted by $\mathbf{z}=\widetilde{\mathbf{f}}(\mathbf{x})$ :

$$
\begin{aligned}
& z_{4}=\tilde{f}_{4}(x), \\
& z_{5}=\tilde{f}_{3}(x) .
\end{aligned}
$$

As it is shown in formula (9), the original affine nonlinear system can be transformed into Brunovsky normalized form:

$$
\begin{aligned}
& \dot{z}_{1}=z_{2}, \\
& \dot{z}_{2}=z_{3}, \\
& \dot{z}_{3}=z_{4}, \\
& \dot{z}_{4}=v_{1}, \\
& \dot{z}_{5}=v_{2} .
\end{aligned}
$$


For the normalized form, control law $\mathbf{v}$ can be obtained by inverse mapping $\mathbf{x}=\widetilde{\mathbf{f}}^{-1}(\mathbf{z})$ from original nonlinear system state feedback control law:

$$
\mathbf{u}=-\mathbf{b}^{-1}(\mathbf{x}) \mathbf{a}(\mathbf{x})+\mathbf{b}^{-1}(\mathbf{x}) \mathbf{v},
$$

where

$$
\begin{gathered}
\mathbf{v}=\left[v_{1}, v_{2}, \ldots, v_{m}\right]^{T}, \\
\mathbf{a}(\mathbf{x})=\left[\widetilde{f}_{1}, \ldots, \widetilde{f}_{m}\right]^{T}, \\
\mathbf{b}(\mathbf{x})=\left[\begin{array}{cccc}
\widetilde{g}_{11} & \widetilde{g}_{21} & \cdots & \widetilde{g}_{m 1} \\
\widetilde{g}_{12} & \widetilde{g}_{22} & \cdots & \widetilde{g}_{m 2} \\
\vdots & \vdots & & \vdots \\
\tilde{g}_{1 m} & \widetilde{g}_{2 m} & \cdots & \widetilde{g}_{m m}
\end{array}\right] .
\end{gathered}
$$

Step 6. Design optimal regulator for the linearized system. After linearization of the system, nonlinear factors have been mapped to the input signal $u$. For inspecting the external dynamic input-to-output performance of the system, this paper completes the controller, at the following two aspects.

(1) Design current tracking controller of the Feedback channel. The purpose of output signals of the system has the ability to more quickly meet the set reference trajectory. Seeing reference trajectory tracking problem as a separate part of the second-order integrator $\mathbf{z}_{\text {ref }}$ requires that poles of the stable linear system meet Hurwitz criterion. Input $\mathbf{v}$ is calculated as

$$
\mathbf{v}=\ddot{\mathbf{z}}_{\text {ref }}+k_{1}\left(\dot{\mathbf{z}}_{\text {ref }}-\dot{\mathbf{z}}\right)+k_{2}\left(\mathbf{z}_{\text {ref }}-\mathbf{z}\right),
$$

where coefficient $k$ satisfied Hurwitz polynomial $s^{2}+k_{1} s+k_{2}=$ 0 .

(2) Optimal controller design. With respect to a particular input signal, examine the quality of output response. During a limited time range, obtain the maximum function through this kind of feedback control system internal electromagnetic torque performance index, so that the process of the output signal goes through transient response to the steady-state. Adopted method called LQR (linear quadratic regulator) is linear quadratic regulator, the given controlled object with a linear state-space system. The objective function is a quadratic function. The aim of LQR design is to make the quadratic objective function $J$ get minimum value which refers to optimal state feedback controller, which is uniquely determined by weight matrices $\mathbf{Q}$ and $\mathbf{R}$, so the choice of the $\mathbf{R}$ and $\mathbf{Q}$ is particularly important to system stability. LQR theory in modern control theory frame is a developed state space design method. Relative to traditional PID regulation mode, LQR can directly obtain the optimal linear feedback control law and easily constitute a closed-loop optimal control.

Theorem 3 (see [27]). For implementing optimal control to a linear system, $\dot{\mathbf{x}}=\mathbf{A} \mathbf{x}+\mathbf{B u}$, if the state space variables are continuous and bounded, there are sufficient and necessary conditions: the corresponding optimal performance index of $\mathbf{u}^{*}=-\mathbf{R}^{-1} \mathbf{B}_{\mathbf{z}}^{\mathrm{T}} \mathbf{P}^{*} \mathbf{x}$ is $J^{*}=(1 / 2) \mathbf{x}^{\mathrm{T}} \mathbf{P} \mathbf{x}$, the symmetric

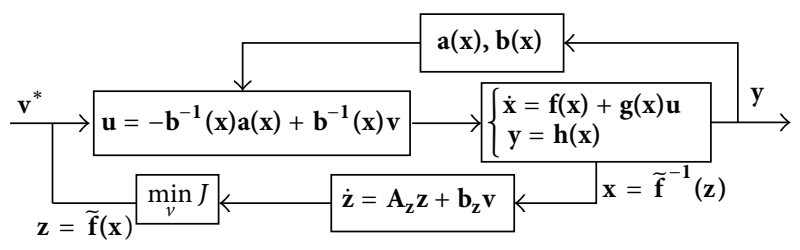

FIGURE 2: General principle diagram of Feedback linearization design.

nonnegative matrix $\mathbf{P}$ satisfies its Riccati equation, and then optimal line $\mathbf{x}^{*}(t)$ is the solution of differential equation $\dot{\mathbf{x}}=$ $\left[\mathbf{A}-\mathbf{B R}^{-1} \mathbf{B}^{\mathrm{T}} \mathbf{P}\right] \mathbf{x}$.

According to Theorem 3, Brunovsky normalized form can be rewritten as $\dot{\mathbf{z}}=\mathbf{A}_{\mathbf{z}} \mathbf{z}+\mathbf{b}_{\mathbf{z}} \mathbf{v}, \mathbf{A}_{\mathbf{z}} \in \mathbb{R}^{n \times n}$, and $\mathbf{b}_{\mathbf{z}} \in \mathbb{R}^{m \times n}$.

Choose the quadratic index $\mathbf{J}$ as shown in formula (32):

$$
\min _{v} \mathbf{J}=\min _{v} \frac{1}{2} \int_{0}^{\infty}\left(\mathbf{z}^{\mathrm{T}} \mathbf{Q} \mathbf{z}+\mathbf{v}^{\mathrm{T}} \mathbf{R} \mathbf{v}\right) d t
$$

According to the principle of optimal control for linear systems, the optimal control law $\mathbf{v}^{*}=-\mathbf{R}^{-1} \mathbf{B}_{\mathbf{z}}^{\mathrm{T}} \mathbf{P}^{*} \mathbf{z}$ is constructed, where $\mathbf{P}^{*}$ is solutions of the Riccati equation:

$$
\mathbf{A}_{\mathbf{z}}^{\mathrm{T}} \mathbf{P}+\mathbf{P A}_{\mathbf{z}}-\mathbf{P B}_{\mathbf{z}} \mathbf{R}^{-1} \mathbf{B}_{\mathbf{z}}^{\mathrm{T}} \mathbf{P}+\mathbf{Q}=0
$$

Thus, obtaining control law $\mathbf{v}$ via the space mappings $Z$ to $X$, the export control law $\mathbf{u}$ of original nonlinear affine systems can be obtained. And the control law $\mathbf{u}$ makes fluctuations of electromagnetic torque kept in the smallest scope, and, under the constraints of performance index $\mathbf{J}$, the physical meaning is optimal value of the original system. In the experiment, it will be seen that the optimal controller can quickly adjust the torque output value near to the state of equilibrium.

Principle diagram of feedback linearization design based on AC induction motor stator flux model is shown in Figure 2.

\section{Stability Discussions of the Designed Controller}

To prove the stability of the complex topology control systems, firstly introduce the relevant principle of small gain stability [16].

Lemma 4 (see [17]). Investigate nonlinear system $\dot{x}=f(x)$, $x \in \mathbb{R}, f(0)=0 ; f(x)$ is local Lipschitz; this system is stable or asymptotically stable at equilibrium point $x=0$, by Lyapunov criterion. Let $V: B_{d} \rightarrow \mathbb{R}$ be a $C^{1}$ class of function; there exist two $\kappa$ functions $\underline{\alpha}(\cdot)$ and $\bar{\alpha}(\cdot)$ in the interval $[0, d)$, which cater to $\underline{\alpha}(\|x\|) \leq V(x) \leq \bar{\alpha}(\|x\|)$, and $\|x\|<d$.

If $(\partial V / \partial x) f(x) \leq 0$, for all $\|x\|<d, \dot{x}=f(x)$ is stable at equilibrium point $x=0$. 
If the interval $[0, d)$ has a $\kappa$ function $\alpha(\cdot)$ which cater to the relationship $(\partial V / \partial x) f(x) \leq-\alpha(\|x\|)$; to all the $\|x\|<d, \dot{x}=$ $f(x)$ is locally asymptotically stable at the equilibrium point $x=0$.

If $d=\infty$ and $\underline{\alpha}(\cdot)$ and $\bar{\alpha}(\cdot)$ of $\underline{\alpha}(\|x\|) \leq V(x) \leq \bar{\alpha}(\|x\|)$ are both $\kappa_{\infty}$ class of function, then $\dot{x}=f(x)$ is globally asymptotical stable at equilibrium point $x=0$.

The definition of input to state stability in modern nonlinear control theory is cited here [14]. For a $C^{1}$ function $V$ : $\mathbb{R}^{n} \rightarrow \mathbb{R}$, if there exist $\kappa_{\infty}$ functions $\alpha(\cdot), \underline{\alpha}(\cdot)$, and $\bar{\alpha}(\cdot)$, there also exists a function $\chi(\cdot)$. They satisfied

$$
\begin{gathered}
\underline{\alpha}(\|x\|) \leq V(x) \leq \bar{\alpha}(\|x\|), \\
\|x\| \geq \chi(\|u\|) \Longrightarrow \frac{\partial V}{\partial x} f(x, u) \leq-\alpha(\|x\|) .
\end{gathered}
$$

For all $x \in \mathbb{R}^{n}$, the function $V$ called an ISS-Lyapunov function of nonlinear systems. And nonlinear system is ISS if and only if ISS-Lyapunov function existed.

Theorem 5 (small gain theorem [28]). For n-order interconnected systems with feedback, if all subsystems $\gamma_{1}(\cdot), \gamma_{2}(\cdot)$, $\ldots, \gamma_{n}(\cdot)$ are ISS, which means for all value $t \geq 0$, to any input $x \in L_{\infty}^{n}$, there must exist $\kappa$ functions as the relative responses that satisfying

$$
\begin{array}{r}
\|x(t)\| \leq \max \left\{\gamma_{1}\left\|x_{1}(\cdot)\right\|_{\infty}, \ldots, \gamma_{n}\left(\left\|x_{2}(\cdot)\right\|_{\infty}\right)\right\}, \\
\lim _{t \rightarrow \infty} \sup \|x(t)\| \leq \gamma_{1}\left(\gamma_{2} \cdots\left(\gamma_{k}\left(\lim _{t \rightarrow \infty} \sup \left\|x_{k}(t)\right\|\right)\right)\right), \\
k=1 \cdots n,
\end{array}
$$

where any complex subsystems $\gamma_{i} \circ \gamma_{i+1}(\cdot), i=0,1, \ldots, n-1$ are simple contract. That is, for all $r>0, \gamma_{1}\left(\gamma_{2}(r)\right)<r$, the whole complex systems are ISS system.

4.1. ISS Stability of the Forward Part of the Nonlinear Coiling System. Whole control system shown in Figure 2 has been discussed in the previous passage which is bounded. For the system as shown as formula (9), it is regarded as a five-dimensional network system. Obviously, the first three equations are directly decoupled with the state variables. Therefore, the other two direct coupling variables are considered nonadditive constant value perturbations. Then, the fifth-order forward coupling system turned into two subsystems interconnected feedback system (33). Firstly, discuss the stability of the forward subsystem. And then discuss the input and output stability of the whole tandem nonlinear control system below.

As one part of the whole asynchronous motor as formula (5), this forward subsystem can be seen as a three-order system with inputs of $d$ - and $q$-axis stator current and with state variables of rotor flux:

$$
\begin{aligned}
& \dot{x}_{1}=\frac{n_{p}^{2} L_{m}}{J}\left(x_{5} x_{2}-x_{4} x_{3}\right)-\frac{n_{p}}{J} T_{L}, \\
& \dot{x}_{2}=-\frac{1}{T_{r}} x_{4}+x_{1} x_{3}+u_{s d}, \\
& \dot{x}_{3}=-\frac{1}{T_{r}} x_{5}-x_{1} x_{2}+u_{s q} .
\end{aligned}
$$

The equilibrium stability of this subsystem is analyzed as below. When the stator current of $q$-axis $x_{5} \neq 0$, solve the subsystem and obtain the equilibrium as

$$
\begin{gathered}
x_{2}=\frac{T_{L} L_{r}}{n_{p} L_{m} x_{5}}+\frac{x_{4} x_{3}}{x_{5}}, \\
x_{1}=\left(\frac{-T_{L} L_{r}}{T_{r} n_{p} L_{m} x_{5}}+\frac{L_{r}}{T_{r}}\right) \times\left(x_{3}\right)^{-1}-\frac{x_{4}}{T_{r} x_{5}}, \\
a x_{3}^{2}+b x_{3}+c=0,
\end{gathered}
$$

where $a=\left(x_{4}^{2} / x_{5}^{2}\right)+1, b=\left(2 T_{L} L_{r} / n_{p} L_{m}\right)\left(x_{4} / x_{5}^{2}\right)-L_{m}\left(x_{4}^{2} / x_{5}^{2}-\right.$ $\left.x_{5}\right)$, and $c=\left(T_{L} L_{r} / n_{p} L_{m} x_{5}\right)^{2}-T_{L} L_{r} x_{4} / n_{p} L_{m} x_{5}$.

Set secular equitation of Jacobian matrix at the equilibrium which is

$$
d^{3}+\lambda_{1} d^{2}+\lambda_{1} d+\lambda_{1}=0
$$

The obtained Eigen values are

$$
\begin{gathered}
\lambda_{1}=\frac{2}{T_{r}} \\
\lambda_{2}=\left(\omega_{1}-x_{1}\right)^{2}+\frac{n_{p}^{2} L_{m}}{J L_{r}}\left(x_{4} x_{2}+x_{5} x_{3}\right)+\frac{1}{T_{r}^{2}}, \\
\lambda_{3}=\frac{n_{p}^{2} L_{m}}{J L_{r}}\left(x_{4} x_{2}+x_{5} x_{3}\right)+\left(\omega_{1}-x_{1}\right)\left(x_{5} x_{2}-x_{4} x_{3}\right) .
\end{gathered}
$$

According to the Routh criterion, necessary and sufficient condition of the stable subsystem is $\lambda_{1}, \lambda_{2}>0, \lambda_{1} \lambda_{2}-\lambda_{3}>0$.

While the motor is running, $T_{L}>0$. Set $\Delta=b^{2}-4 a c$; then the stabilization of the subsystem is as follows.

When $\Delta>0$, all the Eigen values have negative real part. So the subsystem is stable.

When $\Delta=0, x_{4}^{2}+x_{5}^{2}=2 L_{r} T_{L} / n_{p}^{2} L_{m}$; Eigen values are coincident and not positive. So the subsystem is stable too.

When $\Delta<0$, there is no Eigen value of the subsystem. The relevant physical explanations are that there might be certain over load on the motor rotor torque induced by insufficient power supply, and the motor is just in a braking process that the speed response appears as phenomenon of saddle node and bifurcation. As shown in Figure 3, the induction currents in $d$ and $q$ directions are not stable in this special case. 


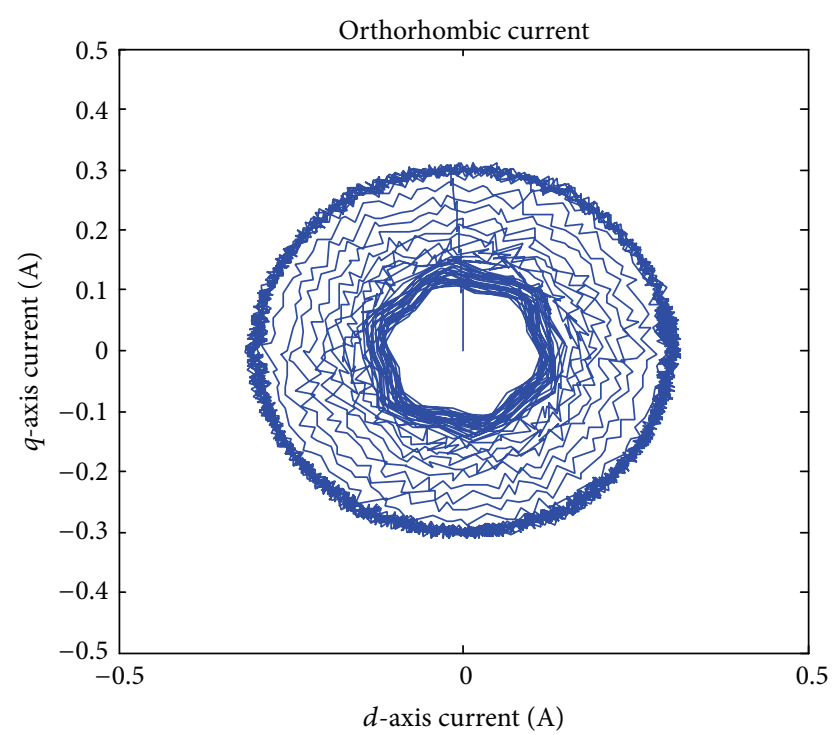

FIGURE 3: Unstable case of induction currents in $d$ and $q$ directions.

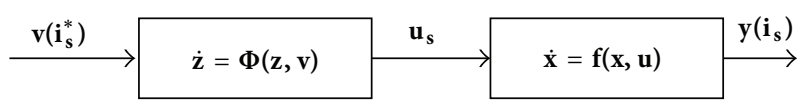

FIGURE 4: Block diagram of the tandem system.

As shown in Figure 3, due to insufficient power supply for impact load, the amplitude of orthorhombic current has fallen down to the ground level. Although the different direction flux was still keeping operation of AC motor, unstable coiling torque might be inevitable, unless an instantaneous implement was given in the input of stator. The theory analysis has practical significance for the asynchronous motor which is applied in some special occasions to avoid unnecessary malfunction. In addition, it should be noted that, under the $d$-axis and $q$-axis currents control, open-loop controlled motor is always stable when the rotor is unloaded.

\subsection{ISS Stability of the Other Part of the Nonlinear Coiling} System in the Whole Tandem System. When the inducted control law obtained by EFL algorithm is put into the whole process, it is equivalent to the original system as shown in formula (9) series with the new control subsystem, as shown in Figure 4.

$\Phi(\mathbf{z}, \mathbf{v})=-a(z) / b(z)+(1 / b(z)) \mathbf{v}$ is calculated law by the method of differential geometry described above. Based on the conclusion of ISS feedback a system is in parallel that $\mathbf{z}$ is a homeomorphism of $\mathbf{x} . \dot{\mathbf{x}}=\mathbf{f}(\mathbf{x}, \mathbf{u})$ can be seen as the forward channel of EFL algorithm and optimal performance computing has not change the original spatial variables which are bounded norm. So following discussion of the ISS character of the tandem input-output system can be reasonably proved.
If a single subsystem is ISS, let system $\dot{\mathbf{x}}=\mathbf{f}(\mathbf{x}, \mathbf{u})$ have ISS dual function $\{\alpha(\cdot), \sigma(\cdot)\}$ and let control law of system $\boldsymbol{\Phi}(\mathbf{z}, \mathbf{v})$ have ISS dual function $\{\beta(\cdot), \zeta(\cdot)\}$. Define function

$$
\widehat{\beta}(\cdot)= \begin{cases}\sigma(\cdot) & \beta(\cdot) \geq \sigma(\cdot), \\ \beta(\cdot) & \beta(\cdot)<\sigma(\cdot),\end{cases}
$$

let $\widehat{\sigma}(\cdot)=\delta \widehat{\beta}(\cdot), 0<\delta<1$, as system $\dot{\mathbf{x}}=\mathbf{f}(\mathbf{x}, \mathbf{u})$ have ISS dual function, set another new dual function $\{\widehat{\alpha}(\cdot), \widehat{\beta}(\cdot)\}$.

Then, according to Lemma 4 and formula (34), there exist positive definite functions $\widehat{u}(x), \widehat{v}(x)$ satisfying

$$
\begin{gathered}
\frac{\partial \hat{u}(x)}{\partial z} \Phi(\mathbf{z}, \mathbf{v}) \leq-\widehat{\beta}(\|\mathbf{z}\|)+\zeta(\|\mathbf{x}\|), \\
\frac{\partial \widehat{v}(x)}{\partial x} \mathbf{f}(\mathbf{x}, \mathbf{u}) \leq-\widehat{\alpha}(\|\mathbf{x}\|)+\delta \widehat{\beta}(\|\mathbf{z}\|) .
\end{gathered}
$$

Let interconnected function $\Phi(x, z)=u(x)+v(x)$; then

$$
\frac{\partial \omega}{\partial x}[\mathbf{f}(\mathbf{x}, \mathbf{u})+\Phi(\mathbf{z}, \mathbf{v})] \leq-\widehat{\alpha}(\|\mathbf{x}\|)+\delta \widehat{\beta}(\|\mathbf{z}\|)+\zeta(\|\mathbf{x}\|) .
$$

Obviously, $₫(x, z)$ ISS-Lyapunov function is a tandem system. To the given system illustrated in Figure 4 according to the ISS stability theory, it is an ISS system:

$$
\begin{gathered}
\dot{x}_{4}=C x_{5}+E x_{2}+G x_{1} x_{3}-F x_{4}-x_{1} x_{5}+G u_{1}, \\
\dot{x}_{5}=-C x_{4}+E x_{3}-G x_{1} x_{2}-F x_{5}+x_{1} x_{4}+G u_{2} .
\end{gathered}
$$

Finally, discuss the stability analysis of the tandem style system. Because the ISS characteristic of interconnected system depends on both the two subsystems, in this case, it is primary to research how the voltage input influences the subsystem stability.

In formula (43), coupling components exist under $d$ and $q$-axis's variables, such as $-x_{1} x_{5}$ and $x_{1} x_{4}$. Meanwhile, inverse potential $-(1 / G) x_{1} x_{2}$ and $(1 / G) x_{1} x_{3}$ From the aspect of control theory, the inverse potential can be seen as adding disturbance. And coupling quantities are independent of this inverse voltage, which can be ignored when analyzing the coupling quantities. This research mainly concerns the problem of decoupling control of the coupling voltage and flux model, as shown in one of the tandem subsystems below:

$$
\begin{aligned}
& u_{d s}=\frac{1}{G}\left(\dot{x}_{4}-x_{1} x_{5}\right)+R_{s} x_{4}, \\
& u_{q s}=\frac{1}{G}\left(\dot{x}_{5}+x_{1} x_{4}\right)+R_{s} x_{5} .
\end{aligned}
$$

There are two inputs and two outputs in this subsystem, stability of which should be discussed in details by linear control theory. For simplifying this model, transfer it to complex number plane. Definition of motor current and voltage under the synchronous coordinate system is

$$
\begin{aligned}
& u_{s}=u_{d s}+j u_{q s}, \\
& i_{s}=x_{4}+j x_{5} .
\end{aligned}
$$


Substitute to formula (44); get the vector from voltage equation of asynchronous motor as

$$
u_{s}=\left(R_{s}+j \frac{1}{G} x_{1}\right) i_{s}+\frac{1}{G} i_{s} .
$$

Then, in the complex plane, this system can be seen as a SISO system. For research, its stability solves this subsystem for acquisition of zeros/poles distribution. With adoption of the nonlinear control law, the current response performance is inevitably influenced by the high-speed coupling voltage in motor winding. In the tandem structure as shown in Figure 4, the transfer function of close loop negative feedback subsystem is

$$
\frac{i_{s}}{i_{s}^{*}}=\frac{a(z) s+b(z)-j x_{1} a(z)}{s^{2}+\left(G R_{s}+G a(z)-j x_{1}\right) s+b(z)-j G x_{1}\left(a(z)+R_{s}\right)} .
$$

There are two poles and one zero in this control system. When the output speed $x_{1}=0$, both poles and zero are located on the negative real axis. So voltages of $d$ - and $q$-axis have no couples. With increasing of the speed, the poles are gradually close to imaginary axis. Although the two poles are not conjugate to each other, the subsystem is always in stable state.

According to formulas (44) and (47), the relationship between stator voltage and current is

$$
\frac{i_{s}}{u_{s}}=\frac{G}{s+G R_{s}+j x_{1}} .
$$

Let ISS-Lyapunov function of this subsystem be $V\left(x_{4}\right)=$ $(1 / 2) x_{4}^{2}$; by calculating the derivative, we have

$$
\begin{aligned}
\dot{V} & =\frac{\partial V}{\partial x} f\left(x_{4}\right) \\
& \leq\left|x_{4}\right|\left[C\left|x_{5}\right|+E\left|x_{2}\right|+G\left|x_{1} x_{3}\right|-F\left|x_{4}\right|-\left|x_{1} x_{5}\right|+G\left|u_{1}\right|\right] .
\end{aligned}
$$

For all $0<\varepsilon<1$, satisfy

$$
(1-\varepsilon)\left|x_{4}\right| \geq \frac{1}{F}\left[C\left|x_{5}\right|+E\left|x_{2}\right|+G\left|x_{1} x_{3}\right|-\left|x_{1} x_{5}\right|+G\left|u_{1}\right|\right] .
$$

Set $\kappa_{\infty}$ function, in order to make $\dot{V} \leq \alpha\left(\left|x_{4}\right|\right)$ established. That is,

$$
\begin{aligned}
\left\|x_{4}\right\| \geq \max \left\{\chi_{1}\left(\left|x_{1}\right|\right), \chi_{2}\left(\left|x_{2}\right|\right),\right. & \\
& \left.\chi_{3}\left(\left|x_{3}\right|\right), \chi_{5}\left(\left|x_{5}\right|\right), \chi_{u_{1}}\left(\left|u_{1}\right|\right)\right\},
\end{aligned}
$$

where $\kappa$ class of functions of state variables is, respectively,

$$
\begin{gathered}
\chi_{1}(r)=\frac{G\left(\left|x_{3}\right|-\left|x_{5}\right|\right) r}{F(1-\varepsilon)}, \\
\chi_{2}(r)=\frac{E r}{F(1-\varepsilon)}, \\
\chi_{3}(r)=\frac{G\left(\left|x_{1}\right|\right) r}{F(1-\varepsilon)}, \\
\chi_{5}(r)=\frac{\left(C-\left|x_{1}\right|\right) r}{F(1-\varepsilon)}, \\
\chi_{u_{1}}(r)=\frac{G r}{F(1-\varepsilon)} .
\end{gathered}
$$

So we can obtain $\underline{\alpha}(r)=\bar{\alpha}(r)=(1 / 2) r^{2}$.

When the norms of state variables in continuous time domain satisfy the condition:

$$
\begin{gathered}
\left\|x_{4}(t)\right\| \leq \max \left\{\chi_{1}\left(\left\|x_{1}\right\|\right), \chi_{2}\left(\left\|x_{2}\right\|\right), \chi_{3}\left(\left\|x_{3}\right\|\right),\right. \\
\left.\chi_{5}\left(\left\|x_{5}\right\|\right), \chi_{u_{1}}\left(\left\|u_{1}\right\|\right)\right\} .
\end{gathered}
$$

To all $t \geq 0$, we have

$$
\begin{aligned}
\lim _{t \rightarrow \infty} \sup \left\|x_{4}(t)\right\| & \\
\leq \max & \left\{\lim _{t \rightarrow \infty} \sup \chi_{1}\left(\left\|x_{1}\right\|\right), \lim _{t \rightarrow \infty} \sup \chi_{2}\left(\left\|x_{2}\right\|\right),\right. \\
& \lim _{t \rightarrow \infty} \sup \chi_{3}\left(\left\|x_{3}\right\|\right), \lim _{t \rightarrow \infty} \sup \chi_{5}\left(\left\|x_{5}\right\|\right), \\
& \left.\lim _{t \rightarrow \infty} \sup \chi_{u_{1}}\left(\left\|u_{1}\right\|\right)\right\} .
\end{aligned}
$$

Conform to formula (35) in Theorem 5 and the upper part of the interconnected system (43) is input subsystem stable state. And then in the lower feedback half interconnected in molecular systems, an appropriate $\kappa$ function can be designed to get $\widehat{\chi}_{1}, \widehat{\chi}_{2}, \widehat{\chi}_{3}, \widehat{\chi}_{4}, \widehat{\chi}_{u_{2}}$.

Finally, checking the small gain condition, to all $r>0$, the entire parallel complex system is satisfied to ISS condition. So get $\chi_{5}\left(\widehat{\chi}_{4} r\right)=2 r / F^{2}(1-\varepsilon)^{2}<r$ and $|F|>\sqrt{2}$. The parallel system is ISS system.

In summary, the system shown in Figure 2 is a tandem which includes interconnected ISS systems, which lies before the new system in Figure 3 as an ISS feedback channel in the entire map. So the whole system with exact feedback linearization controller is an ISS system. Obviously, in the double loops speed regulation system, the current control loop is equal to one-order inertial element. So the element can be designed as an integrator. Because poles of the close loop negative feedback subsystem are real number, the current regulators can realize dynamical decoupling currents, as shown in Figure 5.

As shown in Figure 5, comparing to Figure 3, an instantaneous implement regulation, which was stimulated by the EFL algorithm, was given for the whole interconnected 


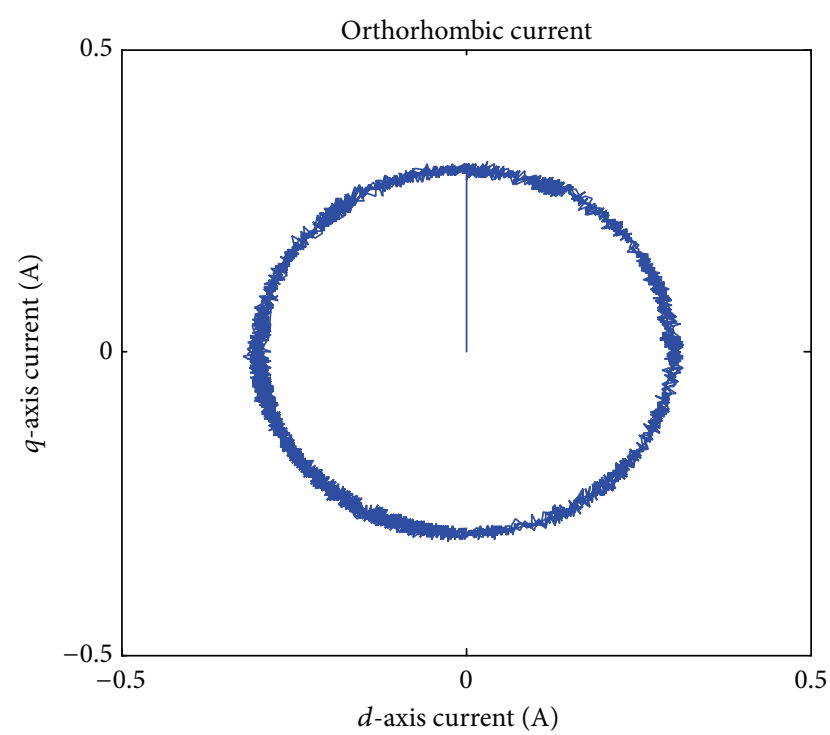

FIGURE 5: Stable case of induction currents in $d$ and $q$ directions.

TABLE 1: Physical and simulation parameters for coiling asynchronous motor.

\begin{tabular}{llc}
\hline VA & Motor power & 33280 \\
Vrms & Voltage & 380 \\
$\mathrm{~Hz}$ & Frequency & 50 \\
$\mathrm{ohm}$ & Stator resistance & 0.436 \\
$\mathrm{ohm}$ & Rotor resistance & 0.818 \\
$\mathrm{H}$ & Stator leakage inductance & 0.002 \\
$\mathrm{H}$ & Rotor leakage inductance & 0.002 \\
$\mathrm{H}$ & Mutual inductance & 0.0691 \\
$\mathrm{~A}$ & Initial current magnitude (phases A/B/C) & $0 / 0 / 0$ \\
$\mathrm{deg}$ & Initial current phase (phases A/B/C) & $0 / 120 / 240$ \\
$\mathrm{Kg} \cdot \mathrm{m}^{2}$ & Inertia & 0.086 \\
$\mathrm{~N} \cdot \mathrm{m} \cdot \mathrm{s}$ & Friction & 0.005 \\
- & Pole pairs & 2 \\
- & Initial slip & 1 \\
$\mathrm{deg}$ & Initial angle & 0 \\
\hline
\end{tabular}

system. Though the vector controller is formally complicated nonlinear model, in respect to common PI controller, additional crossing decoupling factor is included. However, the expression of the control law is too confused to clarify physical significance; the dynamic current control effect displayed in rotor speed response is still swift and fine.

\section{Simulation and Experiment}

5.1. Simulation Research for the Electrical System of $A C$ Coiler. For test availability of the exact feedback linearization approach, a simulation research is made before experiment on physical entities. In this simulation under the stator reference frame test, the physical and simulation parameters for coiling asynchronous motor are specified in Table 1.
TABLE 2: Physical and simulation parameters for controller and inverter.

\begin{tabular}{llc}
\hline $\mathrm{ohm}$ & Rectifier snubber resistance & 0.001 \\
$\mathrm{nF}$ & Rectifier snubber capacitance & 20 \\
$\mathrm{ohm}$ & Diode resistance (on state) & 0.001 \\
$\mathrm{~V}$ & Diode forward voltage & 1.3 \\
$\mathrm{mF}$ & Bus capacitance & 2 \\
$\mathrm{ohm}$ & Braking chopper resistance & 8.2 \\
$\mathrm{~Hz}$ & Braking chopper frequency & 5000 \\
$\mathrm{~V}$ & Braking chopper activation voltage & 400 \\
$\mathrm{~V}$ & Braking chopper shutdown voltage & 380 \\
$\mathrm{ohm}$ & Inverter resistance (on state) & 0.001 \\
$\mathrm{~V}$ & Inverter forward voltage & 0.82 \\
$\mathrm{~ms}$ & Inverter turn-off fall time & 1000 \\
$\mathrm{~ms}$ & Inverter turn-off tail time & 2000 \\
$\mathrm{~F}$ & Snubber capacitance & $\mathrm{Inf}$ \\
$\mathrm{rpm}$ & Speed controller acceleration/deceleration & \pm 2000 \\
- & Proportional gain & 5 \\
- & Integral gain & 10 \\
$\mathrm{~Hz}$ & Speed cutoff frequency & 100 \\
$\mathrm{~ms}$ & Speed controller sampling time & 0.1 \\
$\mathrm{~N} \cdot \mathrm{m}$ & Torque output limit & \pm 5500 \\
$\mathrm{~Wb}$ & Initial motor flux & 0.31 \\
$\mathrm{~Wb}$ & Flux controller limit & \pm 2 \\
$\mathrm{kHz}$ & Maximum switching frequency & 20 \\
\hline
\end{tabular}

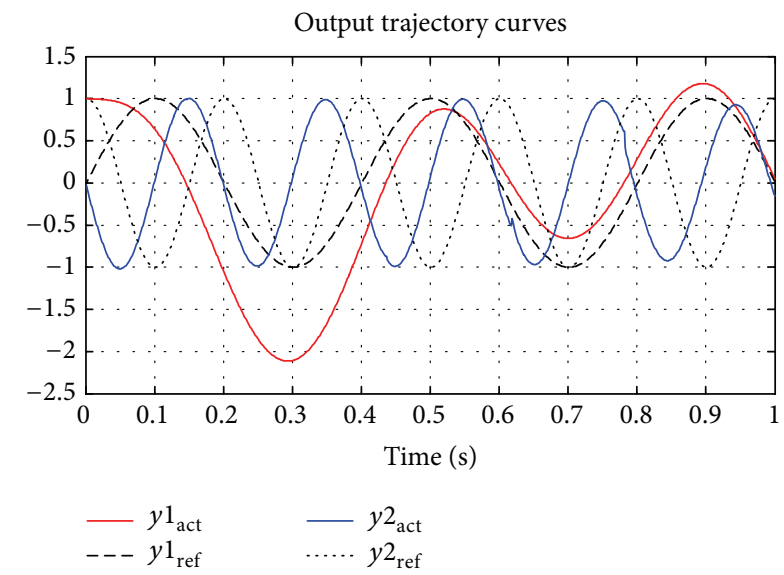

FIGURE 6: Feedback tracking control of two output signals.

The braking chopper, the diode rectifier, the inverter switches parameters, and relevant bus and speed controller parameters are specified in Table 2.

According to formulas (29) and (31), the feedback tracking controller contains the nonlinear control law which is obtained above, a simulation test was made to verify the validity of the theoretical design. Set specified output signal and the initial value as $y 1_{\text {ref }}=\sin (5 \pi t), y 1(0)=1 ; y 2_{\text {ref }}=$ $\cos (10 \pi t), y 2(0)=0$. The simulation result is shown in Figure 6. 
TABLE 3: Main technical parameters of the coiler.

\begin{tabular}{|c|c|c|c|c|c|c|c|c|}
\hline $\begin{array}{l}\text { Product } \\
(\mathrm{mm})\end{array}$ & $\begin{array}{c}\text { Drum } \\
\text { diameter } \\
(\mathrm{mm})\end{array}$ & $\begin{array}{c}\text { Strip } \\
\text { thickness } \\
(\mathrm{mm})\end{array}$ & $\begin{array}{l}\text { Strip width } \\
\quad(\mathrm{mm})\end{array}$ & $\begin{array}{l}\text { Coil diameter } \\
(\mathrm{mm})\end{array}$ & $\begin{array}{c}\text { Volume } \\
\text { weight } \\
(\mathrm{t})\end{array}$ & $\begin{array}{c}\text { Benchmark } \\
\text { tension } \\
(\mathrm{kN})\end{array}$ & $\begin{array}{l}\text { Coiling speed } \\
\qquad(\mathrm{m} / \mathrm{s})\end{array}$ & $\begin{array}{l}\text { Motor power } \\
(\mathrm{kW})\end{array}$ \\
\hline ф510/508 & 510 & $0.2-2$ & $500-1070$ & 1600 & 16 & 15 & $<5$ & 90 \\
\hline ф610 & $570-610$ & $0.3-4.5$ & $550-1250$ & 2000 & 21 & 20 & $<6$ & 90 \\
\hline
\end{tabular}
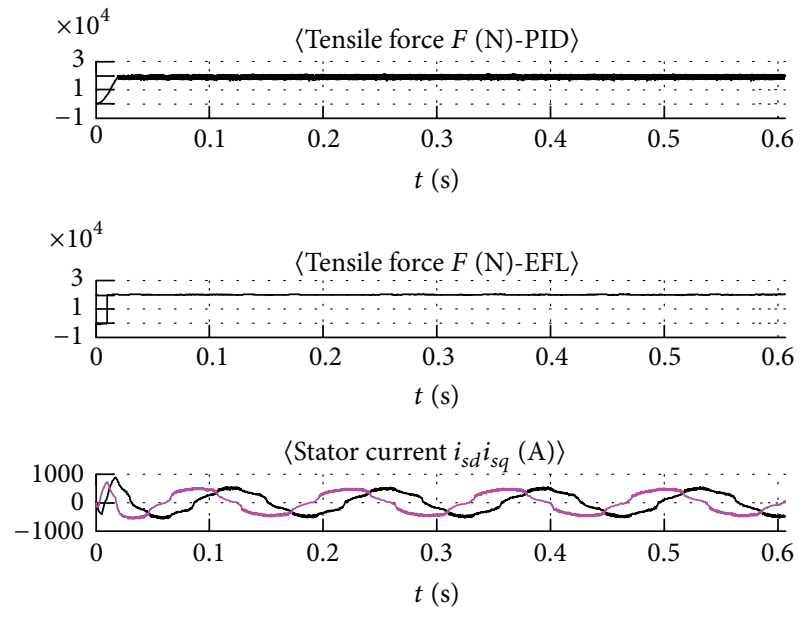

Time offset: 0

FIGURE 7: Tension control of the hot rolling coiler.

Simulation results show that, while the initial per unit value is large, under the action of the nonlinear control law $\mathbf{u}$, the output of the system signal " $y_{\text {act }}$ " can track the reference signal " $y_{\text {ref }}$ " after a brief adjustment process. And through this input-to-output linearization method, the decoupling effect of two output variables is realized.

Substitute all the parameters of Tables 1 and 2 into the models of the coiler as formulas (1) (8). As shown in Figure 7 , through the mechanical torque which have disturbance, simulation results indicated that the input control law obtained from EFL algorithm is characterized by a new complex nonlinear form, which made the current and magnetic flux in the stator change with the stator voltage synchronization more stable than PID algorithm, under the orthogonal rotating coordinates.

The simulation results show that the tension fluctuation is about $10 \%$ by the conventional PID algorithm control, which is bigger than by EFL algorithm. The nonlinear optimal control law after linearization system can make the constant tension control effect is greatly improved that the fluctuation of the tension can be controlled within 3\%. It fully meets the needs of actual production procedures.

5.2. Experiment for Application of This New Nonlinear Controller in AC Coiler System. Based on the simulation result, an experiment for testing the performance of this new nonlinear controller in AC coiler system was carried out. With the assistance of our state key laboratory of plate rolling, the experiments on the reversing mill and coiling control system were initially completed. And the nonlinear drive system containing EFL controller is developed and designed. Hardware configuration of the strip coiling tension control system includes PLC, host computer, photoelectric encoder, the driving device and $\mathrm{AC}$ speed regulating device. The corresponding software of the control system can not only meet the needs of practical production but also have friendly human-machine interface (HMI). The core of the hot rolling control system hardware configuration is shown in Figure 8.

The main parameters of hot rolling coiler AC speed regulating system are as shown in Table 3.

Control system hardware contains the SIMATIC S7-1200 programmable controller of German SIEMENS Company (Inside Profibus), including special coiling software 6dd18431843-0AA1 and standard software package SPW420. The package can be installed on a Windows system and used combining with software STEP7. It is specially designed for $\mathrm{DC} / \mathrm{AC}$ drive option. The configuration and control algorithm of each production line is easily specified in the host computer.

Constant tension control test is carried out under a certain background that a production line of actual hot rolling department fixed the frame parameters of the actual coiler equipment, which has three roll-type hydraulic coilers. The coiling temperature range is $400 \sim 750^{\circ} \mathrm{C}$. Strip thickness range is $1.0 \sim 12.0 \mathrm{~mm}$. Strip width range is $700 \sim 1300 \mathrm{~mm}$. The largest volume weight is 23 tons. The maximum coiling speed is $900 \mathrm{~m} / \mathrm{min}$. The diameter of finished steel coil range is 1200 2000 mm.

Reduction ratio of gear box to the main motor drive system is 2.0 to 1.0. Drum diameter is the maximum size of $770 \mathrm{~mm}$, regular contraction size of $730 \mathrm{~mm}$, and accident contraction size of $722 \mathrm{~mm}$. The forging products' material is heat-resistant stainless steel. Lubrication style belongs to thin oil lubrication (gear box) and concentrated dry lubrication (the remaining parts). Set the simulation time $t=3 \mathrm{~s}$; its simulation parameters are as follows: the rated power of main motor $P_{n}=1000(\mathrm{kVA})$, rated frequency $f_{n}=50(\mathrm{~Hz}), L_{m}=$ $0.251(\mathrm{H}), L_{r}=0.0067(\mathrm{H}), L_{s}=0.0067(\mathrm{H}), R_{s}=1.426$, $R_{r}=1.324, J=0.1\left(\mathrm{~kg} \cdot \mathrm{m}^{2}\right)$, and the number of electrodes $p=$ 2. Experimental results from EFL tracking control algorithm compared to PID control method are shown in Figure 10.

This experiment can test the cases where there are sudden increase and decrease loads on coiler. Parameters of the motor are the same to last step. The experiment results are shown in Figure 9. Figure 9(a) displays the preset torque reference; and Figure 9(b) is contrast of load torque outputs on the rotor under controls of common PID and EFL.

As it is shown in Figure 10, according to the results of the tracking control to asynchronous motor, through 


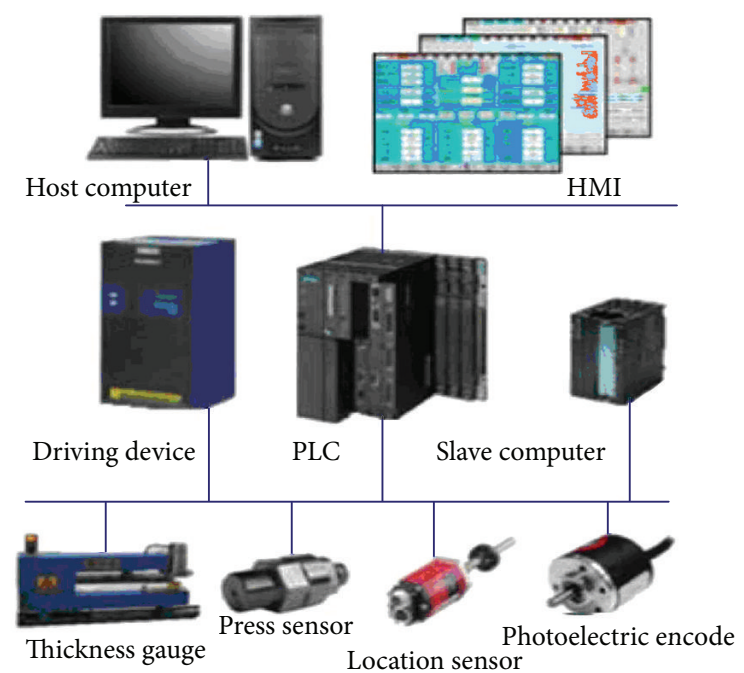

FIGURE 8: The main coiling equipment and hardware configuration of control system.

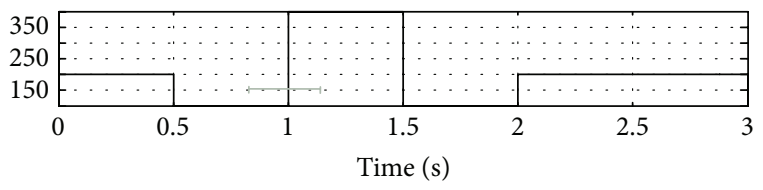

(a) Torque reference $\mathrm{TL} *(\mathrm{~N} * \mathrm{~m})$

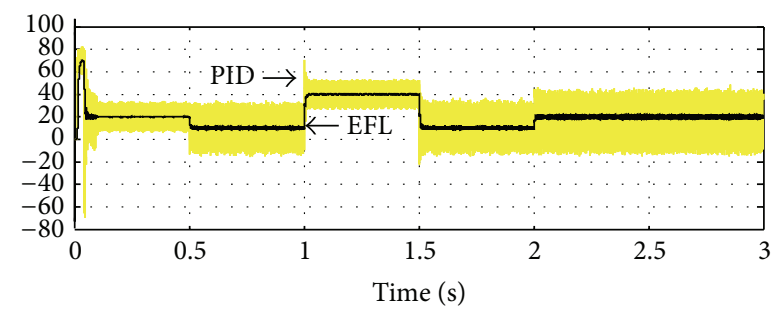

(b) Torque outputs TL $(\mathrm{kN} * \mathrm{~m})$

Figure 9: Comparison of torque outputs under different controllers with respect to the same input.

an optimistic EFL feedback controller, the output of the mechanical torque to the preset reference load torque tracking effect is smoother than PID controller, and the tension deviation is restricted to less than $3 \%$. Electromagnetic torque only had slightly shock during startup process, after the sharp reduction at the moments of $0.5 \mathrm{~s}, 1.5 \mathrm{~s}$; and sudden increment at the moments of $1 \mathrm{~s}, 2 \mathrm{~s}$. With stable effect of the controller, the whole drive system always can quickly track the reference signal. So the system's dynamic performance is good, and overshoot is small too.

Under the same technical conditions, it is clarified shown in comparison Figure 10(b) that responses to dynamically impact load at the moments of biting steel; EFL controller can stabilize fluctuations much better than traditional PID controller. By the faster recognition of nonlinear shocks, the accelerations/decelerations were effectively restricted. The average rotational speed bias was decreased from $0.5 \%$ to $0.1 \%$, which improved the strip yield rate by $5 \%$ and reduced

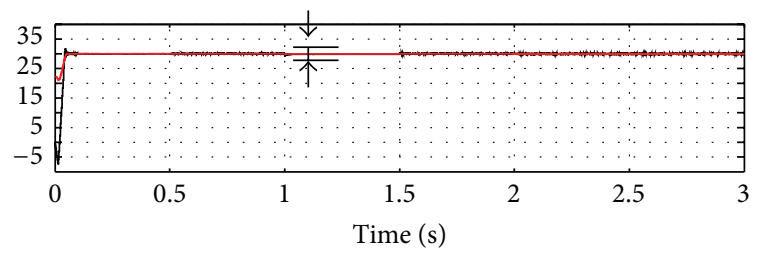

(a) Rotor speed outputs $(r / s)$

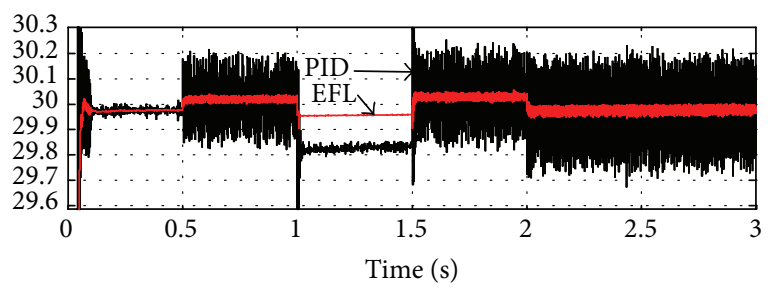

(b) Local amplified rotor speed outputs $(r / s)$

Figure 10: Comparison of rotor speed outputs under different controllers.

the one set of loop bearers and saved much equipment investment.

\section{Conclusion}

In this paper, a new type of dynamic constant tension controller has been proposed for AC motor implemented control of hot rolled plant, and stability of new coiler system with the controller has been proved in theory. The results showed that, for affine nonlinear MIMO system, linearization by EFL algorithm can be realized precisely, as well as for the stator current output dynamic decoupling. This paper gives the detailed theoretical derivation and the process of proof of input to state stability, combined with the actual problems in control engineering. For similar high order controller of complex system design and stability analysis, the idea 
that application of small gain theorem tried to decompose the stability problem of a whole affine nonlinear system into several connected blocks is an effective approach. Experimental results show that the nonlinear controller realizes the fast track of rotating torque output. When impact load has randomly wide range of changes, the nonlinear control law of asynchronous motor control effect is always good; the speed fluctuation is small, the whole system running smoothly. Despite the fact that the control law appears complex nonlinear characteristics, for similar nonlinear mechanical and electrical system to the hot rolling coiler, application of this controller design method can realize high precision speed control of the output signal.

\section{Conflict of Interests}

After confirmation with all authors of this paper entitled "Stability Analysis and Design of a Nonlinear Controller for Hot Rolling Coiler", the authors declare that they have no personal and financial relationships with other people or organizations that can inappropriately influence our research work. There is no question about the validity of this research with professional judgment and no professional or other personal interest of any kind or nature in any service, product, and/or company. And there might no conflict or influencing the position presented in this paper.

\section{Acknowledgments}

This research is supported by National Natural Science Foundation of China (51205018), Research Project of State Key Laboratory of Mechanical System and Vibration (MSV201409), and Fundamental Research Funds for the Central Universities (FRF-TP-14-121A2).

\section{References}

[1] J.-Z. Dong and L. Wang, "Novel control technology of coiler in hot continuous rolling production line," Automation Application, pp. 5-20, 2011.

[2] D. Lihong and C. Xuebo, "Application of Vector control in the cold rolling cleaning coiling inverter system," Metallurgical Industry Automation, vol. 34, no. 3, pp. 69-70, 2010.

[3] D. K. Kori, J. P. Kolhe, and S. E. Talole, "Extended state observer based robust control of wing rock motion," Aerospace Science and Technology, vol. 33, no. 1, pp. 107-117, 2014.

[4] Z.-J. Meng, C.-Z. Sun, and Y.-J. An, "Noninteracting control of multi-input multi-output PMSM based on exact linearization," Journal of Shenyang University of Technology, vol. 32, no. 1, pp. 16-26, 2010.

[5] J. Zhang, Z. Meng, R. Chen, C. Sun, and Y. An, "Decoupling control of PMSM based on exact linearization," in Proceedings of the International Conference on Electronic and Mechanical Engineering and Information Technology (EMEIT '11), vol. 3, pp. 1458-1461, Harbin, China, August 2011.

[6] M. Yin, W. Cong, J. Li et al., "Speed control of permanent magnet synchronous motor based on differential geometry theory and pole placement method," in Proceedings of the IEEE International Conference on Information and Automation (ICIA '10), pp. 2131-2136, 2010.
[7] J. Mao and Y.-Z. Zhou, "Research on the design for permanent magnet synchronous motor drive system based on feedback linearization control," Power Electronics, no. 3, pp. 96-98, 2013.

[8] C.-P. Zhang, F. Li, W.-C. Song, and L.-W. Jiao, "Nonlinear control of induction motors based on direct feedback linearization," Proceedings of the Chinese Society of Electrical Engineering, vol. 23, no. 2, pp. 99-107, 2003.

[9] K. B. Mohanty and M. Singh, "Performance improvement of an induction motor drive using feedback linearization and fuzzy torque compensator," in Proceedings of the Joint International Conference on Power Electronics, Drives and Energy Systems (PEDES '10), pp. 1-7, New Delhi, India, December 2010.

[10] A. Bentaallah, A. Meroufel, A. Bendaoud et al., "Exact linearization of an induction machine with Rotoric flux orientation," Serbian Journal of Electrical Engineering, vol. 5, no. 2, pp. 217227, 2008.

[11] A. T. Dinh, Q. P. Nguyen, and M. D. Le, "A new and effective controller for Inductor Motor drives using Direct-Decoupling Methodology based on exact linearization algorithm and adaptive back stepping technology," in Proceedings of the International Conference on Control Automation and Systems (ICCAS '10), pp. 1941-1945, 2010.

[12] E. D. Sontag, "Smooth stabilization implies coprime factorization," IEEE Transactions on Automatic Control, vol. 34, no. 4, pp. 435-443, 1989.

[13] A. N. Michel and R. K. Miller, Qualitative Analysis of Large Scale Dynamic Systems, Academic Press, New York, NY, USA, 1977.

[14] Z.-P. Jiang, A. R. Teel, and L. Praly, "Small-gain theorem for ISS systems and applications," Mathematics of Control, Signals, and Systems, vol. 7, no. 2, pp. 95-120, 1994.

[15] Z.-P. Jiang, I. M. Mareels, and Y. Wang, "A Lyapunov formulation of the nonlinear small-gain theorem for interconnected ISS systems," Automatica, vol. 32, no. 8, pp. 1211-1215, 1996.

[16] Y. Li, S. Tong, T. Li, and X. Jing, "Adaptive fuzzy control of uncertain stochastic nonlinear systems with unknown dead zone using small-gain approach," Fuzzy Sets and Systems, vol. 235, pp. 1-24, 2014.

[17] I. Karafyllis and Z.-P. Jiang, "A vector small-gain theorem for general non-linear control systems," IMA Journal of Mathematical Control and Information, vol. 28, no. 3, pp. 309-344, 2011.

[18] A. Stern and N. H. Lindner, "Topological quantum computation-from basic concepts to first experiments," Science, vol. 339, no. 6124, pp. 1179-1184, 2013.

[19] W. Heemels, D. P. Borgers, N. van de Wouw et al., "Stability analysis of nonlinear networked control systems with asynchronous communication: a small-gain approach," in Proceedings of the IEEE 52nd Annual Conference on Decision and Control (CDC '13), pp. 4631-4637, 2013.

[20] J. Zhang, X. Huang, Y. Yue, J. Wang, and X. Wang, "Dynamic response of graphene to thermal impulse," Physical Review BCondensed Matter and Materials Physics, vol. 84, no. 23, Article ID 235416, 2011.

[21] J. Zhang, X. Wang, and H. Xie, "Co-existing heat currents in opposite directions in graphene nanoribbons," Physics Letters A, vol. 377, no. 41, pp. 2970-2978, 2013.

[22] Q. Xiong, B. Li, and J. Xu, "GPU-accelerated adaptive particle splitting and merging in SPH," Computer Physics Communications, vol. 184, no. 7, pp. 1701-1707, 2013.

[23] Q. Xiong, L. Deng, W. Wang, and W. Ge, "SPH method for two-fluid modeling of particle-fluid fluidization," Chemical Engineering Science, vol. 66, no. 9, pp. 1859-1865, 2011. 
[24] X. Yang and C.-N. Tong, "Coupling dynamic model and control of chatter in cold rolling," Journal of Dynamic Systems, Measurement and Control, Transactions of the ASME, vol. 134, no. 4, Article ID 041001, 2012.

[25] X. Yang, Q. Li, C.-N. Tong, and Q.-H. Liu, "Vertical vibration model for unsteady lubrication in rolls-strip interface of cold rolling mills," Advances in Mechanical Engineering, vol. 2012, Article ID 734510, 7 pages, 2012.

[26] S. Friedland, S. Gaubert, and L. Han, "Perron-Frobenius theorem for nonnegative multilinear forms and extensions," Linear Algebra and its Applications, vol. 438, no. 2, pp. 738-749, 2013.

[27] F. L. Lewis, H. Zhang, K. Hengster-Movric et al., "Riccati design for synchronization of continuous-time systems," in Cooperative Control of Multi-Agent Systems, pp. 75-105, Springer, London, UK, 2014.

[28] S. Dashkovskiy, M. Kosmykov, and F. Wirth, "A small-gain condition for interconnections of ISS systems with mixed ISS characterizations," IEEE Transactions on Automatic Control, vol. 56, no. 6, pp. 1247-1258, 2011. 


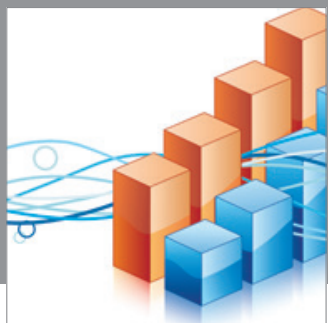

Advances in

Operations Research

mansans

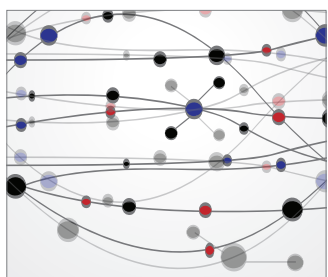

The Scientific World Journal
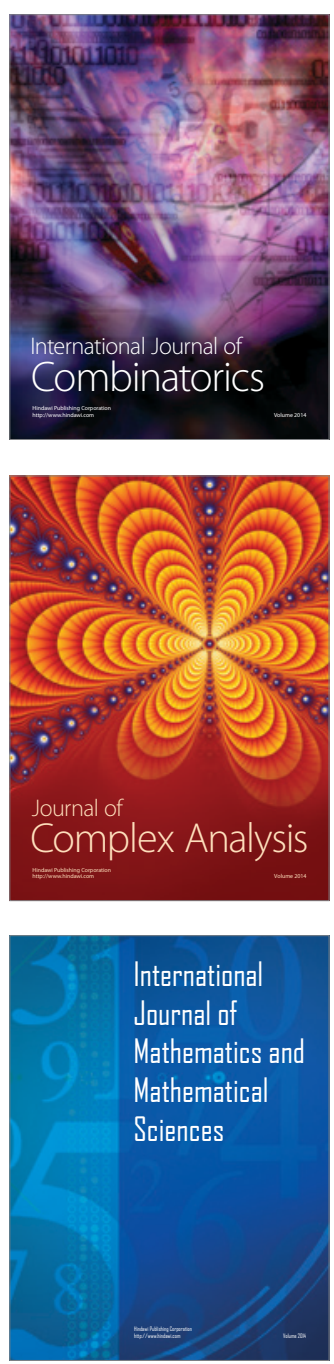
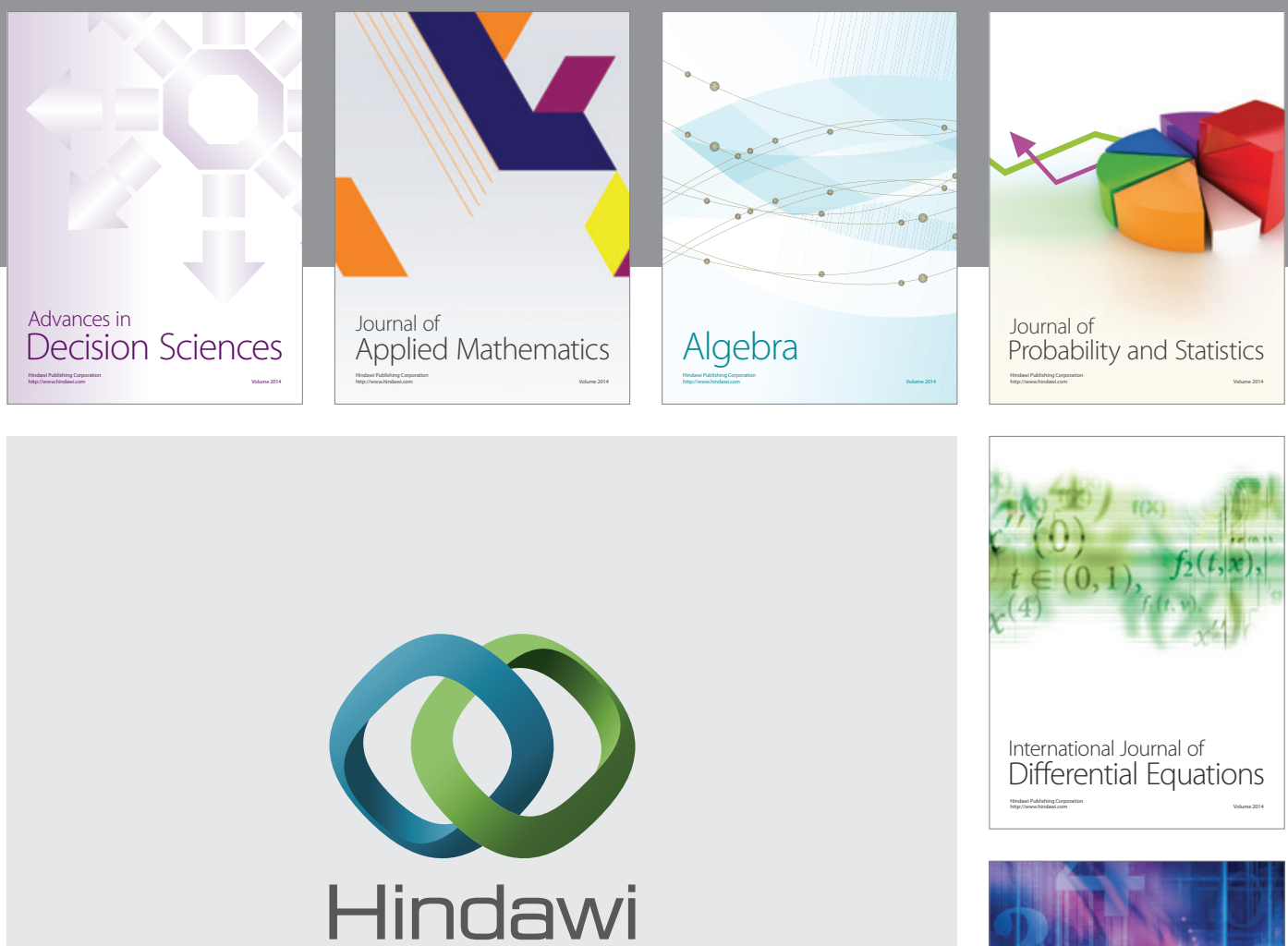

Submit your manuscripts at http://www.hindawi.com
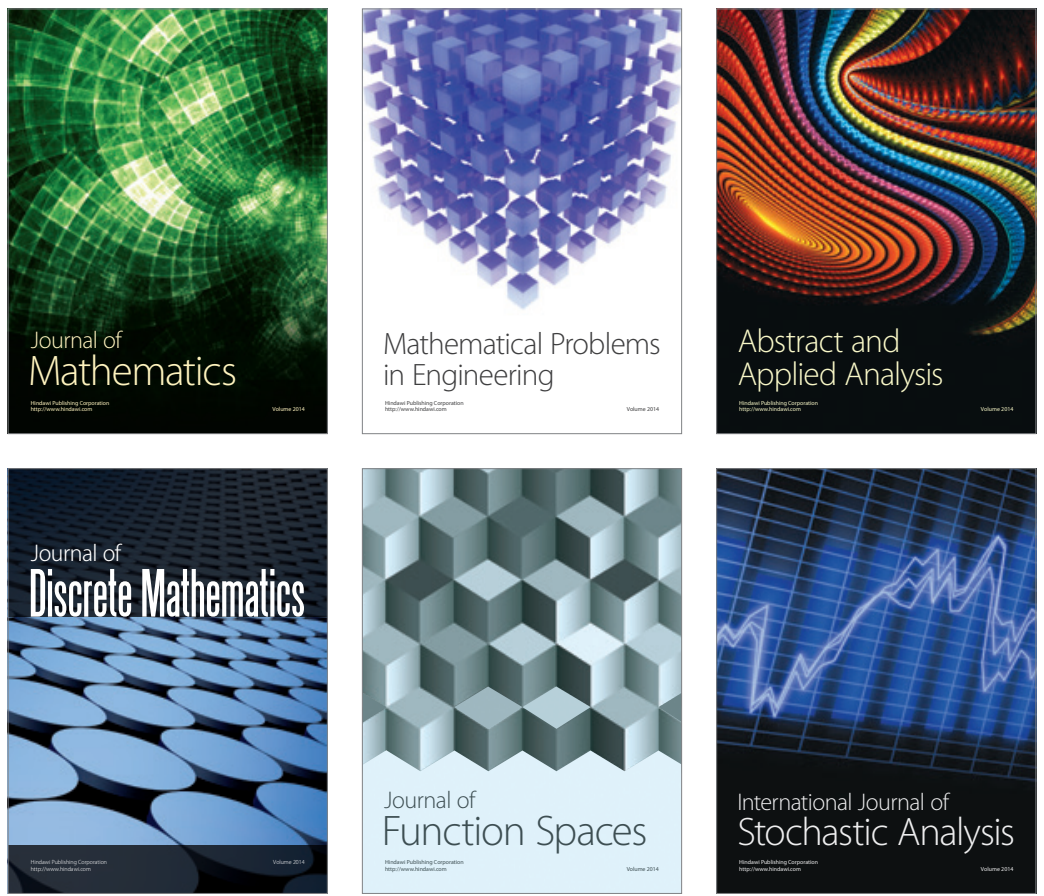

Journal of

Function Spaces

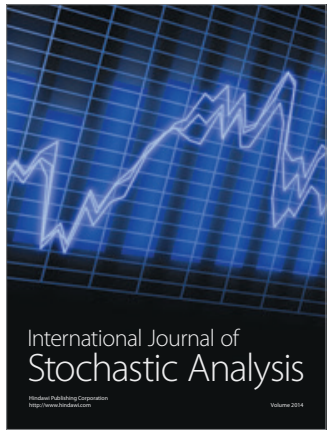

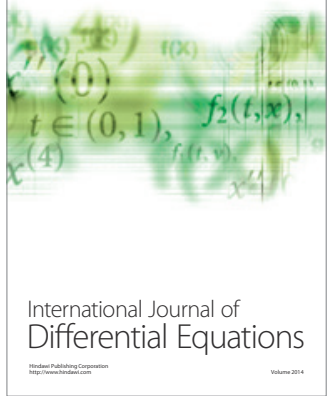
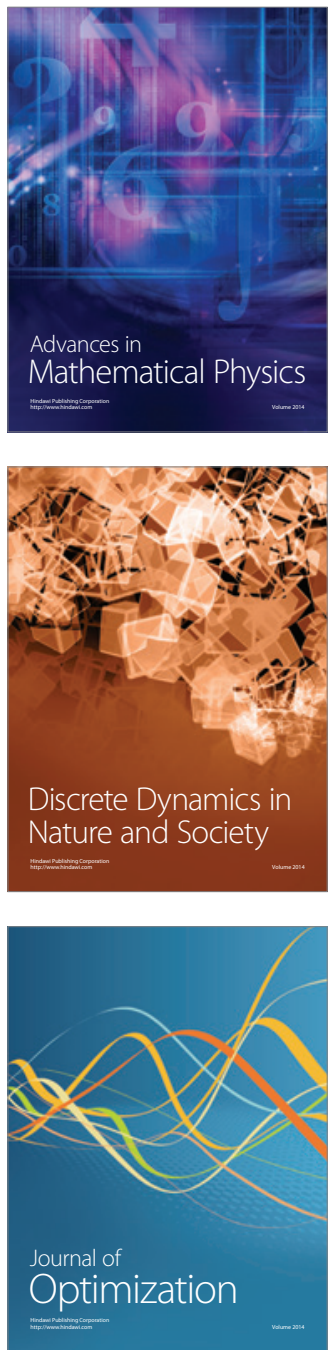